\title{
Princípios básicos de desenho curricular para cursos das profissões da saúde
}

\section{Basic principles to curriculum design on health professions education (HPE)}

Francisco Jose Candido dos Reis ${ }^{2}$, Cacilda da Silva Souza ${ }^{1}$, Valdes Roberto Bollela ${ }^{1}$

\begin{abstract}
RESUMO
Nesta revisão são discutidos os aspectos conceituais e princípios básicos para a construção de currículos para os cursos de gradaução das profissões da saúde. Serão abordados alguns modelos, fundamentos e passos essenciais para a construção de currículos, em paralelo ao contexto das Diretrizes Curriculares Nacionais. No planejamento e na organização dos currículos, é destacada a relevância do comprometimento do professor, aluno e equipe; do emprego de estratégias apropriadas no processo de ensino-aprendizagem e dos instrumentos de avaliação que possam contribuir para a revisão e melhorias do currículo proposto.
\end{abstract}

Palavras-chave: Currículo; Educação médica; Ocupações em saúde.

\section{"Principais Pontos de Interesse"}

1. A definição e comunicação do currículo permite que estudantes, professores e gestores acadêmicos reconheçam suas obrigações em relação ao curso.

2. O currículo é mais que uma lista de atividades e conteúdos. Ele deve informar claramente quais são os resultados esperados com aquela proposta educacional.
3. No processo de desenho curricular é fundamental explicitar qual o propósito do programa educacional, como ele será organizado, as oportunidades de aprendizagem oferecidas pelo programa, e como saberemos se os resultados alcançados foram alcançados ou não.

4. Todas as experiencias vivenciadas pelos estudantes, durante o curso e que os ajudam a alcançar a
1. Departamento de Clínica Médica da Faculdade de Medicina de Ribeirão Preto da Universidade de São Paulo

2. Departamento de Ginecologia e Obstetrícia da Faculdade de Medicina de Ribeirão Preto da Universidade de São Paulo
Correspondência:

Prof. Dr. Francisco Jose Candido dos Reis Departamento de Ginecologia e Obstetrícia, Faculdade de Medicina de Ribeirão Preto. Hospital das Clínicas da FMRP, Campus da USP, 14049-900

E-mail: fjcreis@fmrp.usp.br

Artigo recebido em 22/05/2014 Aprovado para publicação em 19/06/2014 
capacitação profissional são consideradas componentes do currículo.

5. A sustentabilidade do currículo depende de um processo regular de avaliação do próprio programa e da adequação do egresso às necessidades da sociedade.

\section{Introducăo}

Esta revisão tem como objetivo apresentar os princípios básicos para o desenho de currículos e as bases para o desenvolvimento de um currículo na área da saúde. Existem vários fatores que devem ser considerados para o desenho e implementação de um currículo, a saber:

- Teorias de aprendizagem

- Teorias de prática profissional

- Valores sociais

- Expansão constante da base de conhecimento

- Profissionalismo

- Desenvolvimento dos serviços e do sistema de saúde

- Aspectos políticos

- Transparência e responsabilidade.

A escolha do "desenho ou modelo do currículo" não é uma entidade objetiva e exclusivamente racional, mas sim uma construção social, política, acadêmica e profissional ${ }^{1}$. O paradigma da integralidade propõe um equilíbrio entre a excelência técnica e a relevância social, e busca valorizar as metodologias pedagógicas desenvolvidas na área da educação para aperfeiçoar o processo ensino-aprendizagem. A formação profissional, a produção de conhecimento e a prestação de serviços pelas instituições formadoras são então inseridas em contexto de relevância social, ou seja, a responsabilidade do ensino perante a sociedade.

A aprovação em 2001 das Diretrizes Curriculares Nacionais (DCN) dos cursos de graduação em medicina, pode ser considerada consequência de uma mobilização transformadora dos educadores da área da saúde no país e reflexo das tendências internacionais que propõem inovações na formação dos profissionais de saúde ${ }^{2}$. Nesta conjuntura, a maioria dos cursos de graduação da área da saúde no Brasil passou a enfrentar seus dilemas, entre os quais o de conciliar a necessidade de incorporação de um volume crescente de novos conhecimentos e tecnologias e atender às demandas sociais geradas pelas peculiari- dades e desigualdades do país. A formação exclusiva ou preponderante nos hospitais de alta complexidade e a carência de profissionais com formação baseada em competências resultam em discrepâncias entre o perfil do profissional que a sociedade deseja e o egresso dos aparelhos formadores da área da saúde. Diante desta questão, na virada do século XXI, os cursos de graduação da área da saúde no Brasil buscaram revisar as suas diretrizes curriculares.

São crescentes as expectativas de mudança do modelo de currículos nos cursos de graduação, que formatados em "grades" conferem excessiva rigidez advindas, em grande parte, de fixação detalhada nos conteúdos das disciplinas ${ }^{3}$. Em boa medida, as recomendações explícitas nas Diretrizes Curriculares Nacionais (DCN) de 2001 têm sido uma excelente referencia para desenho e revisão dos currículos no Brasil.

O planejamento e o desenvolvimento de currículos são matérias prioritárias na agenda dos cursos de graduação, pós-graduação e de educação médica continuada. O modelo de educação em que o professor restringe seus ensinamentos apenas aos temas do seu próprio interesse, ou o treinamento prático, aos pacientes internados em hospitais terciários, tem sido questionado. Outra situação que tem se tornado cada vez menos frequente é a do professor que desenha/ revê o currículo de seu curso/disciplina baseado apenas na sua própria e prévia experiência de aprendizado acadêmico, ou seja, a reprodução de práticas de ensino, sem a reflexão sobre as mudanças na sua área de atuação, no perfil dos estudantes e na prática profissional. Assim, não existe dúvida sobre o papel crucial do planejamento cuidadoso do currículo para que o processo de ensino/aprendizagem tenha sucesso.

\section{Conceito de currículo}

Currículo é mais que uma lista de atividades ou um conjunto de conteúdos. O currículo deve cuidar de todos os aspectos relevantes para um programa de ensino. Ele deve informar claramente quais são os resultados esperados com a proposta educacional, bem como sobre a intenção dos professores e quais as escolhas e os caminhos a serem tomados para se alcançar estes resultados esperados (Figura 1). Vale ressaltar alguns conceitos agregados ao currículo, e que geralmente são debatidos. O currículo "declarado", é o currículo formal e que está impresso nos documentos da instituição. O currículo "ensinado" é o 
resultado da "leitura" que os professores fazem e aquilo que executam, a partir do que está determinado no currículo declarado, ou seja, é aquilo que é executado pelos professores, o que acontece efetivamente na prática. O currículo "oculto" é definido como tudo aquilo que os estudantes aprendem, mas que não faz parte das atividades previstas no currículo declarado, e apesar de não ser formalmente ensinado pelos professores do curso e/ou não estarem previstos na carga horária do curso, resulta em aprendizado para os estudantes. Finalmente, existe o conceito de currículo "aprendido", que estaria relacionado a tudo aquilo que foi possível aos estudantes aprenderem, independentemente se nas atividades formais ou informais de aprendizado.

\section{Construção do currículo}

Com intuito de facilitar a compreensão das bases da estruturação e da implementação curricular, foram propostos vários modelos que estabelecem "eta- pas" para o desenho de currículos efetivos no cumprimento das suas finalidades. Discutimos a seguir dois modelos de construção curricular. O modelo proposto por Harden (1986) e outro modelo baseado em competências, que foi proposto pelo Accreditation Council for Graduate Medical Education-ACGME $(2000)^{4,5}$.

Na Tabela 1 são apresentados os 10 passos na estruturação do currículo segundo Harden, e em seguida são comentados, de forma resumida, os principais pontos da proposta do ACGME e sua relação com as Diretrizes Curriculares Nacionais para a Área da Saúde, de 2001.

Idealmente, deve-se estabelecer a priori os resultados de aprendizagem (learning outcomes) de uma experiência educacional, o que será a base norteadora do currículo. Neste modelo, os resultados de aprendizagem esperados devem ser previamente definidos e informados, e determinam a tomada de decisões sobre o currículo. Esta visão contrasta com modelos anteriores de desenho de currículos, centrados na ex-

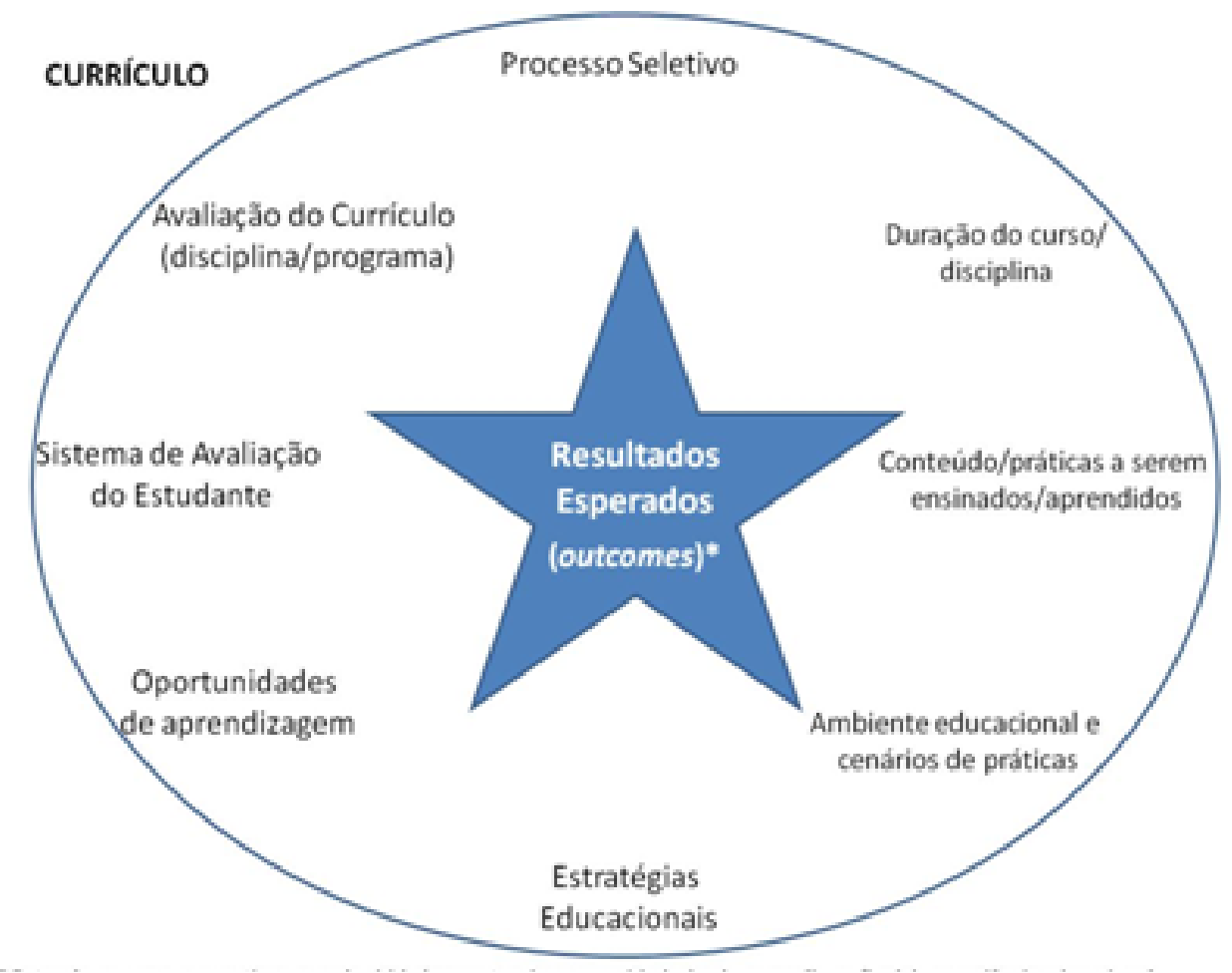

Figura 1: Componentes de um currículo, a começar pelos resultados esperados (estrela) e todos os recursos mobilizados e envolvidos para que sejam alcançados.

* Outcomes ou resultados esperados são as competências e capacidades esperados do aprendiz, ao final da experiência educacional. 
Tabela 1. Dez passos para a estruturação do currículo

1. Identificar as necessidades do aprendiz

2. Estabelecer os resultados da aprendizagem

3. Concordar com o conteúdo

4. Organizar o conteúdo

5. Decidir a estratégia educacional

6. Decidir e ensinar métodos

7. Preparação da avaliação

8. Comunicação sobre o currículo

9. Promoção de ambientes educacionais adequados

10. Gestão do currículo

periência de ensinar e nos métodos de ensino. Nesta nova proposta, o foco principal deve ser direcionado para os resultados da aprendizagem.

$\mathrm{Na}$ área da saúde é fundamental buscar o desenvolvimento de habilidades e atitudes entre os estudantes, no mesmo grau de importância que o da aquisição de conhecimento teórico. Neste sentido, o conteúdo do currículo deve necessariamente concordar com a prática profissional e ser pautado pelas necessidades da sociedade para a qual o futuro profissional se destina. A organização do conteúdo depende do modelo de currículo adotado pelo curso. Um currículo mais tradicional determina a sequencia de aprendizado que se inicia pelas ciências básicas, incluindo anatomia, fisiologia e bioquímica, seguindo para as ciências aplicadas, como patologia, microbiologia e epidemiologia (ciclo básico). Somente após essa fase, e que se dá o aprendizado relacionado à prática profissionalizante. A crítica comum a essa abordagem é que os estudantes podem não perceber o que é relevante para a sua prática profissional futura, e a aquisição dos conhecimentos estaria dissociada da compreensão de como e onde seriam úteis na solução de problemas reais. A desconexão temporal e a falta da contextualização propiciariam um aprendizado pouco efetivo. Nas propostas atuais, o conteúdo curricular deve estar integrado e permanentemente presente na cabeça do estudante, o que permitiria ao estudante vivenciar a prática profissional desde o inicio de sua formação.

Existem propostas de integração curricular, do tipo do currículo integrado vertical, em que os estudantes são introduzidos à prática junto com as ciências básicas numa fase precoce do programa. Os estudantes continuam a ver as ciências básicas aplicadas à prática nos anos subsequentes.

Todas as estratégias educacionais devem ser explicitadas no currículo, e para defini-las os professores deverão considerar alguns princípios norteadores de práticas educacionais mais modernas, bem definidas nas DCNs e que na literatura são referidas por meio do acrônimo SPICES, apresentado na Tabela 2. Tais aspectos devem ser sempre ponderados por quem elabora o currículo de qualquer experiência educacional, seja uma simples aula, uma disciplina, um estágio ou um curso inteiro.

O modelo SPICES sugere uma continuidade entre os dois polos na organização das atividades curriculares, e que cada gestor do currículo deva trabalhar com as possibilidades de transitar entre cada um dos polos. Uma breve descrição das seis dimensões propostas no modelo SPICES pode ser vista na Tabela 3.

Tabela 2: Modelo SPICES de estratégias educacionais

\begin{tabular}{|c|c|c|c|}
\hline \multicolumn{4}{|c|}{ O Modelo SPICES prevê um continuum entre os dois polos de cada tema } \\
\hline $\mathbf{S}$ & Centrado no estudante & $\leftarrow--\cdot-\cdot------\rightarrow$ & Centrado no professor \\
\hline $\mathbf{P}$ & Baseado em problemas & $\leftarrow---------\rightarrow$ & Orientado por informações \\
\hline I & Integrado & $\leftarrow---------\longrightarrow$ & Focado nas disciplinas/especialidades \\
\hline $\mathbf{C}$ & Baseado na comunidade & $\leftarrow------------\longrightarrow$ & Baseado nos Hospitais \\
\hline $\mathbf{E}$ & Conduzido eletivamente & $\leftarrow------------\longrightarrow$ & Uniforme \\
\hline $\mathbf{S}$ & Sistemático & $\leftarrow------------\rightarrow$ & Oportunístico \\
\hline
\end{tabular}

* S: student centered; P: problem based; I: integrated; C: community-based education; E: electives; S: systematic approach of learning. 


\section{Tabela 3: Descritivo das seis dimensões propostas no modelo SPICES}

\begin{tabular}{|c|c|c|}
\hline \multicolumn{3}{|r|}{ Modelo SPICES * descritivo } \\
\hline$\overline{\mathbf{S}}$ & Centrado no estudante & $\begin{array}{l}\text { A responsabilidade pelo seu processo educacional deve ser do estudante. Es- } \\
\text { ses estudantes vão estabelecer diferentes tempos para o seu estudo, a depender } \\
\text { das suas necessidades. }\end{array}$ \\
\hline $\mathbf{P}$ & Baseado em problemas & $\begin{array}{l}\text { Tem sido uma abordagem sedutora na educação médica, pois oferece uma atra- } \\
\text { tiva combinação de pragmatismo e idealismo. Pragmatismo no sentido de que } \\
\text { a aprendizagem é vista como uma importante fonte de motivação e satisfação: } \\
\text { o idealismo que está em consonância com as teorias atuais da educação. }\end{array}$ \\
\hline $\mathbf{I}$ & Integrado & $\begin{array}{l}\text { Em currículos plenamente integrados os estudantes teriam a visão da prática } \\
\text { na perspectiva de outros profissionais. }\end{array}$ \\
\hline C & Baseado na comunidade & $\begin{array}{l}\text { Existem inúmeros e sólidos argumentos que suportam o planejamento de um } \\
\text { currículo baseado mais na comunidade do que em hospitais. Muitos currículos } \\
\text { já preveem atividades fora do hospital ou clínicas escola desde o primeiro ano } \\
\text { do curso de graduação. }\end{array}$ \\
\hline $\mathbf{E}$ & Conduzido eletivamente & $\begin{array}{l}\text { Os programas eletivos estão bem estabelecidos nas escolas a sua importância } \\
\text { tem aumentado na atividade educacional. Pode ser visto como um componente } \\
\text { selecionado pelo estudante no currículo. Promove oportunidades para que o } \\
\text { estudante escolha áreas de seu interesse e desenvolva habilidades em auto ava- } \\
\text { liação, avaliação crítica e manejo do tempo. }\end{array}$ \\
\hline $\mathbf{S}$ & Sistemático & $\begin{array}{l}\text { A complexidade crescente das práticas médicas especializadas e a a necessida- } \\
\text { de de assegurar que todos os alunos tenham experiências de aprendizado com- } \\
\text { paráveis faz com que tenhamos de considerar uma oferta de aprendizado em } \\
\text { ambientes simulados e não apenas na prática dos cenários reais, pois as vezes } \\
\text { nem todos os estudantes terão a mesma oportunidade de aprender algo impor- } \\
\text { tante, se o currículo prevê apenas uma abordagem oportunistica (se tiver opor- } \\
\text { tunidade ele aprende). }\end{array}$ \\
\hline
\end{tabular}

* S: student centered; P: problem based; I: integrated; C: community-based education; E: electives; S: systematic approach of learning.

\section{Metodologias de ensino}

O currículo também deve abordar os métodos de ensino a serem utilizados no curso/disciplina. As diversas estratégias de ensino podem ser organizadas ao longo do curso para facilitar o processo de aprendizagem. As diversas estratégias têm particularidades que podem ser aproveitadas para maximizar o aproveitamento nas diversas situações e cenários. Mas é fundamental que sejam aplicadas de forma apropriada e planejada, o que muitas vezes implica em capacitação do corpo docente.

A aula teórica e as estratégias de sala de aula continuam sendo ferramentas poderosas para abordagem de ensino em grandes grupos, se usadas adequadamente. Existem técnicas para dinamizar aulas teóricas, inclusive para plateia numerosa (vide tema: TBL).

O trabalho em pequenos grupos facilita a interação entre estudantes e torna possível aprendizado colaborativo com os estudantes aprendendo uns com os outros. Trabalho em pequenos grupos é parte importante do PBL. Nas profissões da saúde uma parte significativa da aprendizagem ocorre nos cenários de pratica. A aprendizagem através da pratica possibilita além da aquisição de conhecimentos e habilidades, o desenvolvimento de atitudes essenciais para o adequado desenvolvimento profissional futuro. O aprendizado independente pode trazer uma importante contribuição. Os estudantes desenvolvem a capacidade de trabalhar por conta própria e assumem a responsabilidade por sua própria aprendizagem. 
O processo de avaliação do estudante é um componente chave do currículo. Já está bem documentado o impacto significativo que a avaliação do estudante causa na aprendizagem. Este tópico deverá ser abordado de modo específico e capítulos específicos.

É fundamental que todos os interessados conheçam a proposta educacional em profundidade, e é responsabilidade dos gestores e professores comunicar efetivamente os detalhes da proposta aos estudantes, corpo administrativo e à sociedade.

O ambiente educacional é um aspecto chave para a efetivação do currículo. É menos tangível que o conteúdo estudado, o método de ensino ou a avaliação, no entanto, de igual importância. Se o ambiente é mais competitivo que colaborativo, será difícil desenvolver no estudante o espírito de equipe.

O modelo de gestão influencia todo o desenvolvimento do currículo. Deve-se buscar um modelo colegiado e de alta responsabilidade. Todos os atores do processo de aprendizagem precisam estar representados, as decisões precisam ser compartilhadas. Por outro lado, é fundamental que todos estejam cientes de sua responsabilidade. Neste modelo de gestão, há ampla possibilidade de se antecipar dificuldades e trabalhar soluções antes da ocorrência dos problemas. Uma apropriada articulação do processo transmite segurança aos professores e estudantes. Há ainda mais garantias da continuidade do processo à medida que os seus participantes são substituídos, e evita mudanças bruscas e rupturas que pouco contribuem para os avanços necessários.

\section{Curriculo baseado em competên- cias}

As seguir será apresentada sumariamente, como exemplo, uma abordagem de desenho curricular em seis passos proposta pelo $\mathrm{ACGME}^{5,6}$.

Currículo baseado em competências é aquele que deixa claro quais as competências que se espera do aprendiz e deve ser descrito em termos de objetivos de aprendizagem específicos (resultados esperados). Cada objetivo deve estar relacionado com um plano que descreva "como" ele será alcançado e "como" essa aquisição será medida (avaliada).

As condições essenciais para o desenvolvimento de um currículo baseado em competências são: um grupo de interessados e uma base conceitual e teórica de determinado tema, aliadas à uma proposta de tra- balho de aprendizagem baseada na troca de conhecimentos, experiências e da prática (aprender fazendo). É importante entender que o currículo do curso, como um todo, é composto por um conjunto de propostas curriculares para cada unidade de ensino/aprendizagem (disciplinas, módulos, estágios, rodízios do internato, etc.) ao longo dos seis anos da formação médica. Para cada unidade de aprendizagem, será necessário escrever a proposta curricular que não deve ser entendida apenas como o plano de ensino ou ementa da disciplina/estágio.

A estratégia proposta a seguir tem como base o guia do facilitador para o desenvolvimento de um currículo baseado em competências da Accreditation Council for Graduate Medical Education (ACGME), de 2006, que, apesar de ter foco na estruturação de currículos médicos para a especialização (residência médica), oferece a descrição de abordagens, que pela clareza e praticidade, são úteis para o trabalho com os currículos dos cursos de graduação.

Para a realização do exercício será proveitosa a identificação de uma unidade de ensino/aprendizagem constante do seu currículo, ou uma experiência educacional sobre a qual se pretende desenvolver um currículo baseado em competências e os desfechos esperados (outcomes). O guia traz orientações passo a passo, para facilitar o desenvolvimento de um currículo baseado em competências, que servirá de modelo para futuras construções ou revisões curriculares do seu curso. Nossa expectativa é que essa proposta de estruturação curricular possa ajudá-lo a estruturar um currículo de graduação, sem ter a pretensão de ser algo definitivo e/ou completo. Existe uma grande margem para as adequações e complementações que cada disciplina, módulo ou rodízio requerem dentro do contexto específico da escola médica e de sua inserção na comunidade.

O planejamento curricular é uma ferramenta muito útil para deixar claro para os estudantes e professores o que se pretende e o que se espera com a experiência educacional proposta. São seis os passos necessários para executar um planejamento curricular baseado em competências estão apresentados na Tabela 4 .

Neste modelo busca-se estabelecer a priori o que se espera que os aprendizes (estudantes, residentes, pós-graduandos) incorporem desta experiência educacional específica e garantir que estejam alinhadas com as necessidades de saúde da população a que este futuro profissional servirá. 
Tabela 4: Passo a passo do desenho curricular segundo modelo do ACGME, 2006

\section{Passo a Passo do Desenho Curricular - ACGME}

Passo 1. Necessidades: Avaliação do que será necessário aprender nesta experiência educacional e que são relevantes para a prática profissional.

Passo 2. Identificar as competências que podem ser desenvolvidas e/ou adquiridas na experiência educacional.

Passo 3. Descrever na essência o que significa ser competente ou ter alcançado os resultados esperados (outcomes) ao final da experiência educacional através de objetivos educacionais.

Passo 4. Garantir oportunidades de aprendizagem.

Passo 5. Determinar os métodos de avaliação do estudante.

Passo 6. Determinar como a experiência educacional será avaliada e melhorada.

Por exemplo, quais das competências gerais das DCN poderiam ser desenvolvidas por estudantes que concluírem a disciplina de Ginecologia e Obstetrícia (GO) do quarto ano, onde estão previstas atividades teóricas e práticas na unidade básica de saúde e na maternidade do município. É fundamental que os objetivos específicos e as competências reflitam trajetórias que levem na direção dos resultados esperados para aquela atividade proposta.

É absolutamente necessário que exista um alinhamento entre os objetivos de aprendizagem esperados e as oportunidades de aprendizagem que a experiência educacional lhes proporcionará, além de estabelecer as metodologias de ensino que serão utilizadas (aulas teóricas, seminários, clube de revista, práticas de laboratórios, simulações, prática clínica, estudo individual, etc..). Suponha que no exemplo anterior, foi estabelecido, como objetivos de aprendi- zagem da disciplina de GO, que o estudante deverá ser capaz de realizar a anamnese, exame físico da gestante, anotar os achados no cartão de pré-natal e prover as orientações necessárias para uma gestante em consulta de pré-natal normal. A partir deste ponto, estamos obrigados, enquanto responsáveis pela experiência educacional, a criar oportunidades em número suficiente para todos os estudantes alcançarem esses objetivos e, ao final, sejam capazes de conduzir de forma adequada uma consulta de pré-natal em paciente com gestação normal. Se esse exemplo parece óbvio, um programa de disciplina que inclua entre seus objetivos, por exemplo, que o estudante seja capaz de proceder com proficiência uma punção venosa central ao final do estágio de urgência e emergência, na sua organização deve estar garantida a oportunidade a todos os estudantes de realizarem tal procedimento. Se esta condição não for atendida, a exclusão deste objetivo do programa do estágio deve ser considerada.

Determinar os métodos de avaliação do estudante que serão empregados para conferir o desenvolvimento da competência ou a capacidade para desempenhar uma atividade esperada. Informar ao estudante como ele/ela será avaliado.

Informar aos professores e estudantes como será feito o acompanhamento e avaliação do estágio/ disciplina e como o feedback dos envolvidos poderá contribuir para a revisão e melhoria daquela experiência educacional no futuro.

Seguramente, a partir de um plano preliminar serão necessários vários movimentos, revisões e adequações para a construção de um currículo contemplando as propostas pretendidas. Ressalta-se, que na sua construção e execução, o currículo deva continuar envolvendo toda a equipe e dinamicamente passará por transformações em resposta às mudanças da sociedade, do conhecimento e das tecnologias, que possivelmente refletirão na formação dos profissionais das áreas da saúde.

\section{ABSTRACT:}

In this review we present the concepts and the basic principles for curriculum design in health professions education (HPE), and present some curriculum design models as well as the essential steps for those who are facing this challenge. All the discussion brings the perspective of National Curriculum Guidelines for HPE in Brazil. It is highlighted the importance of the stakeholders' engagement in this process and the correct use of appropriate strategies of teaching-learning, students' assessment tools that can contribute to the implementation/review and improvement of curriculum in HPE courses.

Keywords: Curriculum; medical education; health professions education. 


\section{Referencias Bibliográficas}

1. Grant J. Principles of curriculum design. Understanding Medical Education: Wiley-Blackwell; 2010. p. 1-15.

2. Almeida MJD, Campos JJB, Turini B, Nicoletto SCS, Pereira LA, Rezende LR, et al. Implantação das Diretrizes Curriculares Nacionais na graduação em Medicina no Paraná. Rev Bras Educ Méd. 2007;31:156-65.

3. Maranhão EA. A construção coletiva das Diretrizes Curriculares Nacionais dos Cursos de Graduação da Saúde: uma contribuição para o Sistema Único de Saúde. In: Almeida, MJ. Diretrizes Curriculares Nacionais para os Cursos Universitários da Área da Saúde. Rede Unida. Londrina. 2003.
4. Harden RM. Ten questions to ask when planning a course or curriculum. Med Educ. 1986;20:356-65.

5. ACGME/ABMS. Outcome project (2006). Accreditation Council for Graduate Medical Education and American Board of Medical Specialties. http://cores33webs.mede.uic.edu/gmenext/ui/portal/external/gc_about.htm. Acesso em 18 de fevereiro de 2014.

6. Bollela VR, Machado JLM. Internato baseado em competências: Bridging the gaps. Editora Medvance. 1를 Ed. Belo Horizonte, 2010. 